\title{
Imaging observations of the equatorward limit of midlatitude traveling ionospheric disturbances
}

\author{
K. Shiokawa ${ }^{1}$, Y. Otsuka ${ }^{1}$, M. K. Ejiri ${ }^{1}$, Y. Sahai ${ }^{1}$, T. $\operatorname{Kadota}^{1}$, C. Ihara $^{1}$, T. Ogawa ${ }^{1}$, K. Igarashi ${ }^{2}$, S. Miyazaki ${ }^{3}$, and A. Saito ${ }^{4}$ \\ ${ }^{1}$ Solar-Terrestrial Environment Laboratory, Nagoya University, Toyokawa 442-8507, Japan \\ ${ }^{2}$ Communications Research Laboratory, 4-2-1 Nukui-Kita, Koganei, Tokyo 184-8795, Japan \\ ${ }^{3}$ Geographical Survey Institute, 1-Kitasato, Tsukuba, Ibaraki 305-0811, Japan \\ ${ }^{4}$ Graduate School of Science, Kyoto University, Kitashirakawa-Oiwakecho, Sakyo-ku, Kyoto 606-8502, Japan
}

(Received October 31, 2000; Revised July 2, 2001; Accepted July 25, 2001)

\begin{abstract}
This paper reports the first attempt to observe the equatorward limit of medium-scale traveling ionospheric disturbances (TIDs) in the middle latitudes. The TIDs usually propagate southwestward in the northern hemisphere. An all-sky cooled-CCD imager measured 630-nm airglow at a southern island of Japan, Okinawa $\left(26.9^{\circ} \mathrm{N}, 128.3^{\circ} \mathrm{E}\right.$, geomagnetic latitude $(\mathrm{MLAT})=17.0^{\circ}$ ), during the FRONT-2 campaign of August 4-15, 1999. The TIDs were detected at the mainland of Japan $\left(\sim 21^{\circ}-36^{\circ}\right.$ MLAT) by the total electron content (TEC) observations of more than 1000 GPS receivers. In the August 4 event, the TIDs moving southwestward was seen only in the northern sky of Okinawa as a depletion band in the 630-nm airglow images. In the August 6 event, the TIDs were not seen in the 630-nm images at Okinawa, although weak TID activity was observed by the GPS network at the mainland of Japan. The TEC data also showed weakening of the TID activity below $18^{\circ}$ MLAT. Based on these observations, we suggest that there is a possible limit of medium-scale TID propagation around $\sim 18^{\circ}$ MLAT.
\end{abstract}

\section{Introduction}

Traveling ionospheric disturbances (TIDs) are a manifestation of the interactions between the ionized atmosphere and atmospheric gravity waves (Hines, 1964; Hooke, 1968; Perkins, 1973; Francis, 1974; Hamza, 1999). The new technique of wide-angle airglow imaging gives the two-dimensional structure and direction of motion of the TIDs. Mendillo et al. (1997) and Garcia et al. (2000) have shown midlatitude TIDs as band-like structures in the OI $630-\mathrm{nm}$ airglow images, on the basis of observations from Arecibo, Puerto Rico (geomagnetic latitude $($ MLAT $)=28.8^{\circ}$ ). The band-like TID structures have wavelengths of 50-500 km and move southwestward with velocities of $50-170 \mathrm{~m} / \mathrm{s}$. Taylor et al. (1998) reported similar airglow-band structures moving southwestward, using observations from Yamagawa, Japan (21.4 MLAT).

The other new technique, measurement of total electron content (TEC) by more than 1000 receivers of the Global Positioning Satellite (GPS) signal over Japan, has been directed by the Geographical Survey Institute, Japan. This GPS-TEC network also gives the two-dimensional band structure of TIDs moving southwestward over Japan $\left(\sim 21^{\circ}-\right.$ $36^{\circ}$ MLAT) (Saito et al., 1998). Saito et al. (2001) and Kubota et al. (2000) have used the network of observations of 630-nm airglow and GPS-TEC over the Japanese islands during the first F-region Radio and Optical measurement of Nighttime TIDs (FRONT) campaign, to show that the TIDs travel more than $1000 \mathrm{~km}$ in the southwestward direction

Copy right (C) The Society of Geomagnetism and Earth, Planetary and Space Sciences (SGEPSS); The Seismological Society of Japan; The Volcanological Society of Japan; The Geodetic Society of Japan; The Japanese Society for Planetary Sciences. and the enhancement of TEC in TIDs corresponds to the enhancement of the 630-nm airglow intensity. However, since the network of GPS receivers and airglow imagers was limited mostly to the mainland of Japan, the equatorward limit of the midlatitude TID propagation was not identified by these network observations.

In order to detect the equatorward limit of the southwestward-moving TIDs, the second FRONT campaign (FRONT-2) was carried out on August 4-15, 1999, by adding a new $630-\mathrm{nm}$ airglow measurement site at Okinawa (17.0 $0^{\circ}$ MLAT). In this short paper, we report two TID events that were detected during this campaign period. In one event, the TIDs reached the Okinawa latitude, and in the other, the TIDs were not detected by the Okinawa imager.

\section{Observations}

Figure 1 shows the location of the stations used in this paper. An all-sky imager was sited at Hedo, Okinawa $\left(26.9^{\circ} \mathrm{N}\right.$, $\left.128.3^{\circ} \mathrm{E}, 17.0^{\circ} \mathrm{MLAT}\right)$ during the FRONT-2 campaign of August 4-15, 1999. The northern edge of the imager fieldof-view (FOV) reaches to the mainland of Japan, where the network of GPS receivers is available. The southern edge of the FOV reaches a geographic latitude of $19^{\circ}\left(\sim 9^{\circ}\right.$ MLAT $)$. Ionosonde data are available from Kokubunji $\left(35.7^{\circ} \mathrm{N}\right.$, $\left.139.5^{\circ} \mathrm{E}, 26.5^{\circ} \mathrm{MLAT}\right)$ and Okinawa $\left(26.3^{\circ} \mathrm{N}, 127.8^{\circ} \mathrm{E}\right.$, $16.3^{\circ} \mathrm{MLAT}$ ) for the campaign. All the magnetic latitudes and dip angles (inclinations) used in this paper were calculated using the IGRF-95 model for an epoch time of January 1,2000 .

During the campaign, 630-nm airglow observations were also carried out in the mainland of Japan (Rikubetsu, 


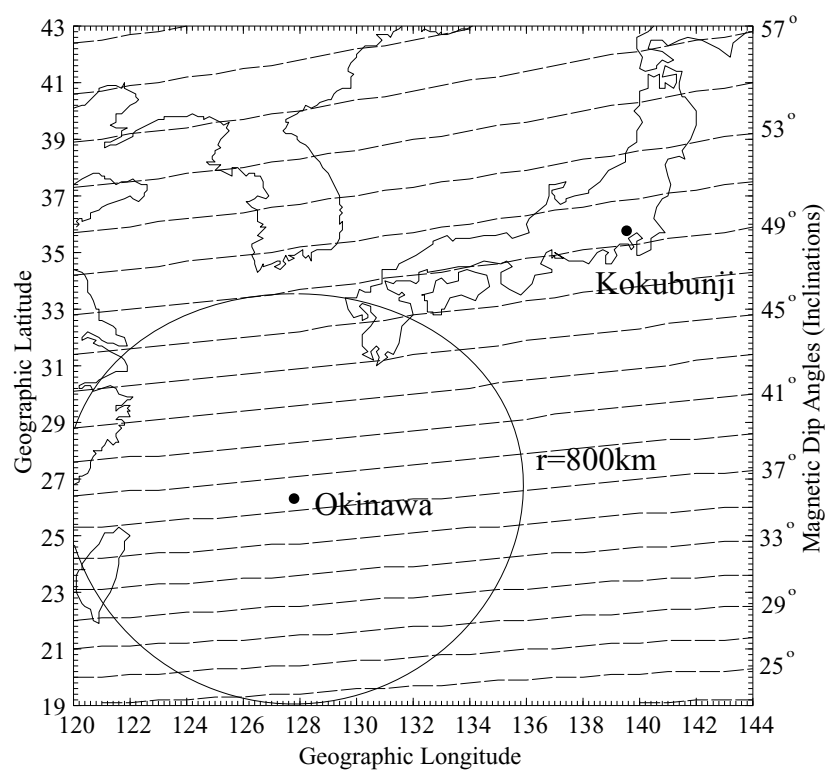

Fig. 1. Map showing the locations of stations. The airglow data shown in Fig. 2 were obtained at Okinawa $\left(26.9^{\circ} \mathrm{N}, 128.3^{\circ} \mathrm{E}, 17.0^{\circ}\right.$ MLAT). The ionogram data shown in Fig. 5 were obtained at Kokubunji $\left(35.7^{\circ} \mathrm{N}, 139.5^{\circ} \mathrm{E}, 26.5^{\circ} \mathrm{MLAT}\right)$ and Okinawa. The circle indicates the field-of-view of an all-sky imager at Okinawa with a horizontal radius of $800 \mathrm{~km}$.
MLAT $=34.7^{\circ} \mathrm{N} ;$ Zao, $29.0^{\circ} \mathrm{N}$; Shigaraki, 25.4 $\left.{ }^{\circ} \mathrm{N}\right)$. However, simultaneous data from these sites were not obtained due to sky conditions. Because of humid weather during the tropical summer in Okinawa, the nights of August 4 and 6 were almost the only intervals in which clear sky conditions were available. The $K p$ indices for $1200-1500$ UT were $1+$ and 4- for August 4 and 6, 1999, respectively.

\subsection{Airglow measurements}

The all-sky imagers that we used for observations were developed as part of the Optical Mesosphere Thermosphere Imagers (OMTIs) (Shiokawa et al., 1999). They have five 3-inch filters on each wheel, a fish-eye lens which has a FOV of $180^{\circ}$, and a back-illuminated cooled-CCD camera with $512 \times 512$ pixels. Details of the all-sky imagers are given by Shiokawa et al. (2000a). During the FRONT-2 campaign, the airglow images at the wavelength of $630 \mathrm{~nm}$ (OI) were taken every $5.5 \mathrm{~min}$ with an exposure time of 2.75 min. Images at wavelengths of $557.7 \mathrm{~nm}$ (OI, $1.75 \mathrm{~min}$ exposure), 720-910 nm (OH, $15 \mathrm{~s}$ exposure), and $572.5 \mathrm{~nm}$ (sky background) were also obtained by rotating the filter wheel. Local time in Okinawa is 8.5 hours ahead of UT $(1530 \mathrm{UT}=0000 \mathrm{LT})$.

Figures 2(a) and (b) show the 630-nm airglow images obtained on August 4 (1324-1402 UT = 2154-2232 LT) and 6 (1148-1337 UT = 2018-2207 LT), 1999, respectively For both images, the Milky Way (Galaxy) can be seen from
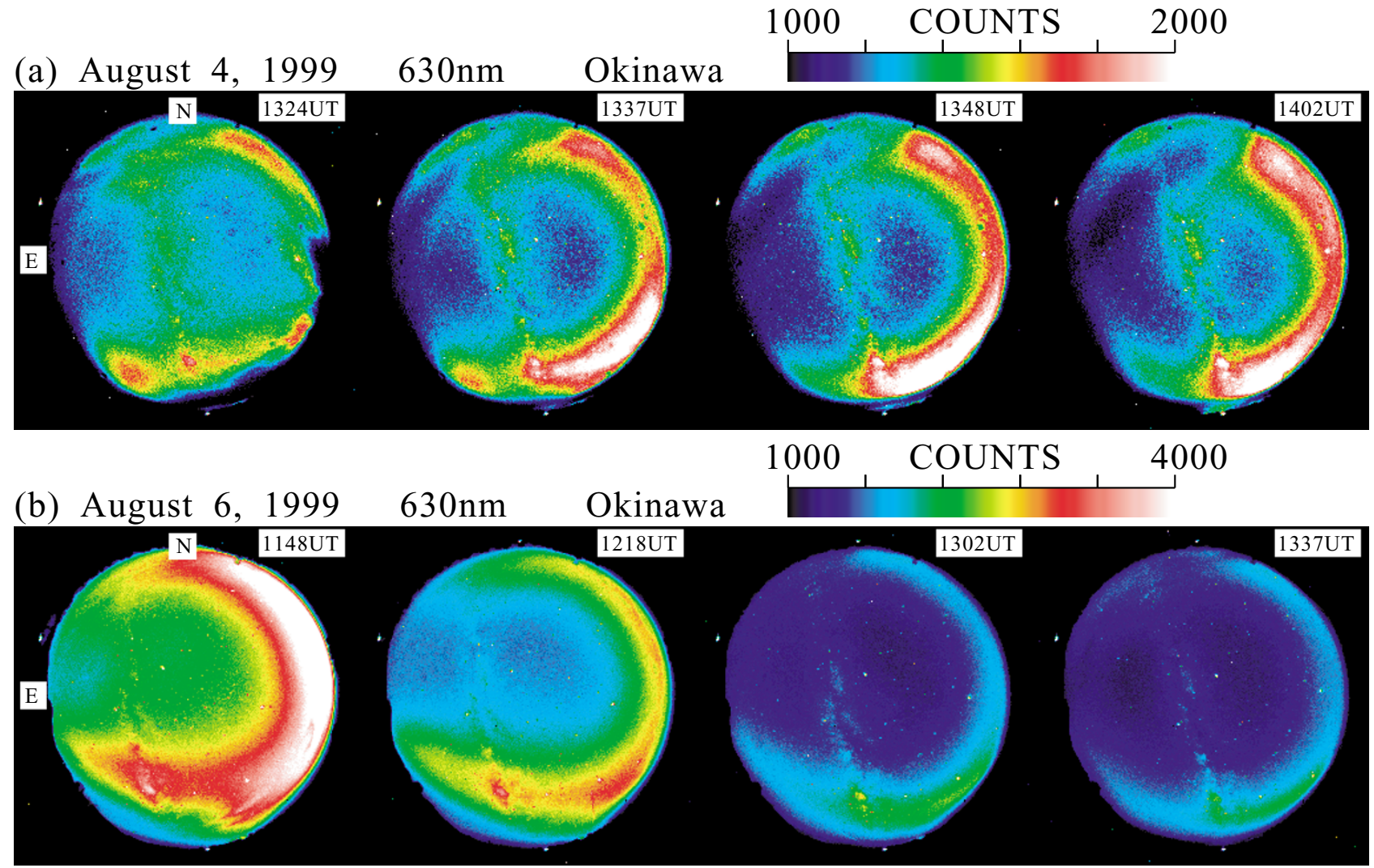

Fig. 2. Airglow images at a wavelength of $630 \mathrm{~nm}$ obtained at Hedo, Okinawa, during the FRONT-2 campaign at (a) 1324-1402 UT (2154-2232 LT) on August 4 and (b) 1148-1337 UT (2018-2207 LT) on August 6, 1999. North is upward, and east is to the left. The Milky Way (Galaxy) is seen in these images from NNE to SSW. The intense emission in the south in Fig. 2(a) is city light. A clear airglow depletion band moving southwestward is seen in the northeast in Fig. 2(a). An intense airglow band, probably corresponding to the equatorial ionospheric anomaly, is seen in Fig. 2(b). 
(a)
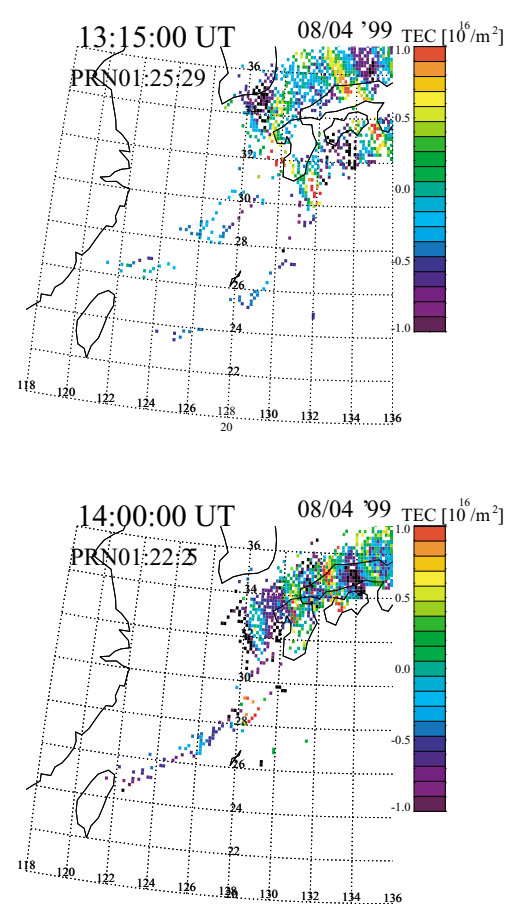

(b)
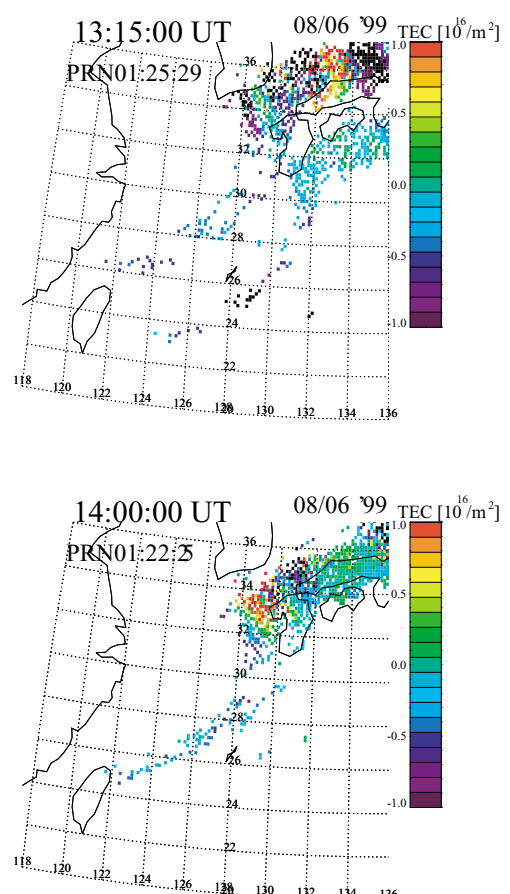

\section{August 4, 1999}
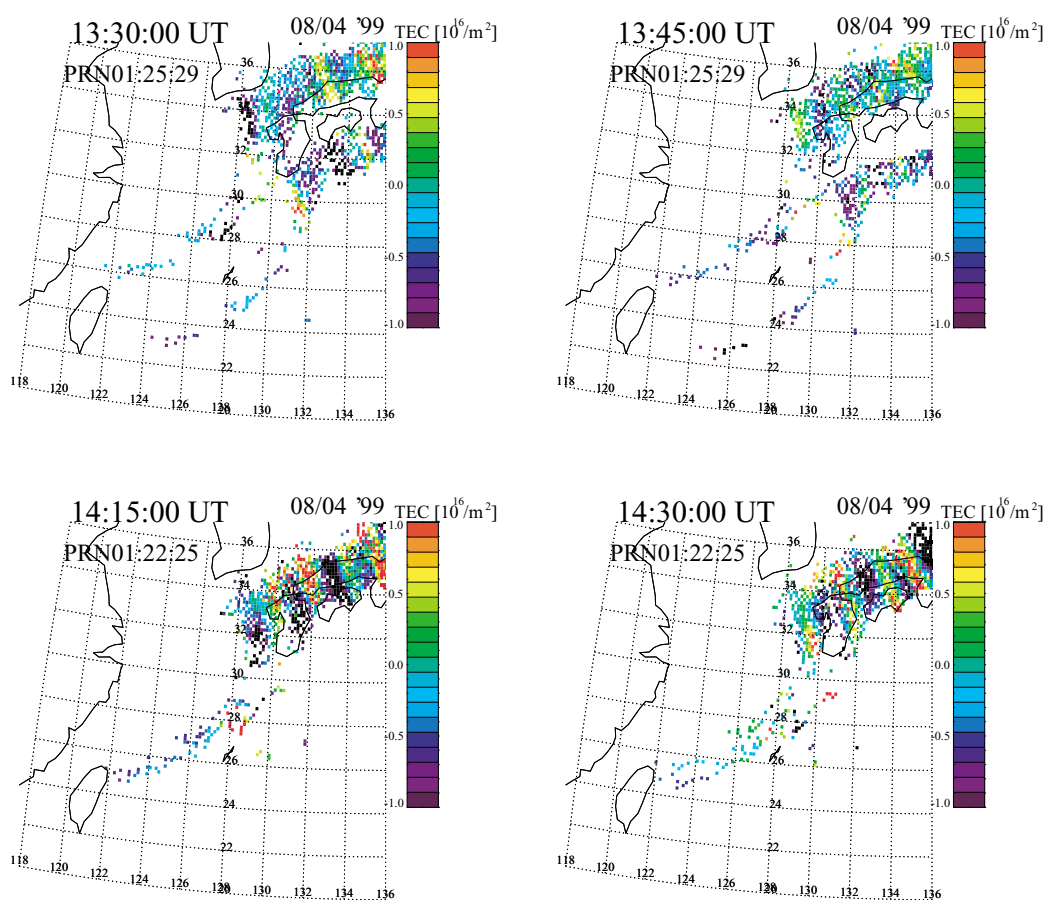

\section{August 6, 1999}
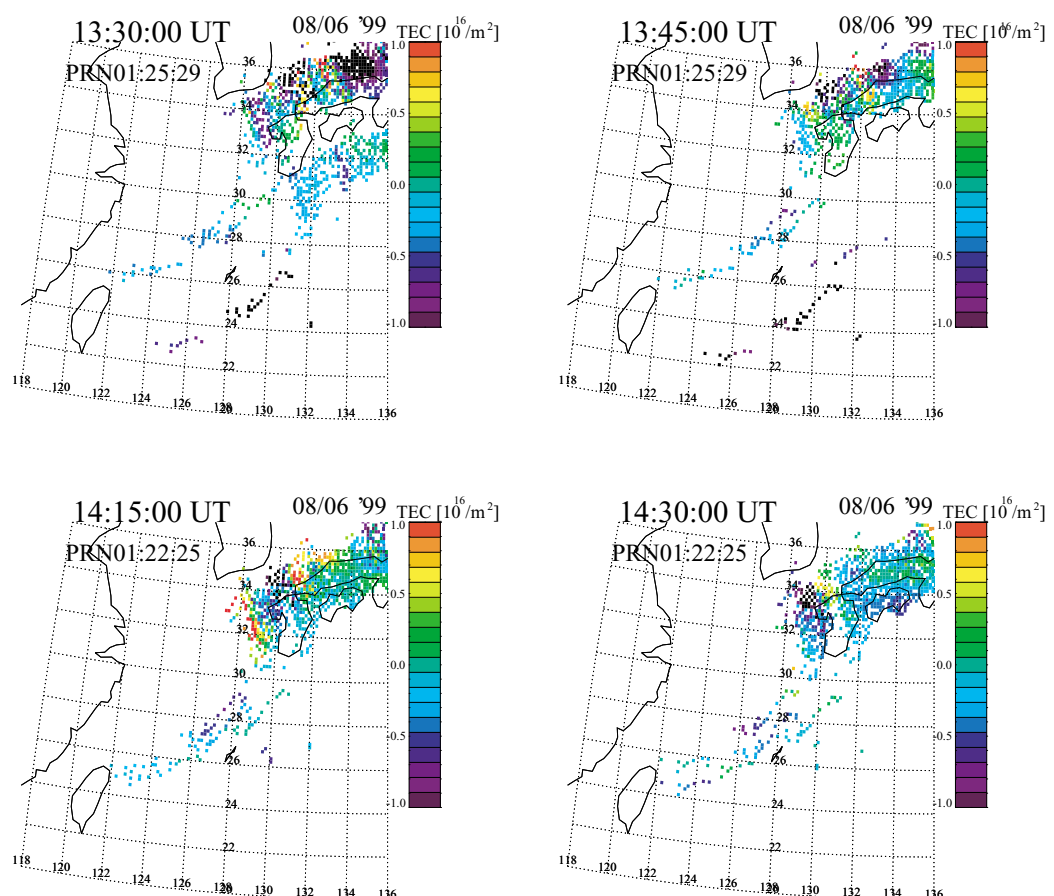

Fig. 3. Two-dimensional maps of TEC variations obtained by more than 1000 GPS receivers in Japan during the FRONT-2 campaign of (a) August 4, and (b) August 6, 1999. The TEC values are shown as differences from the running average over 2 hours. The locations of the TEC values are those mapped at an altitude of $250 \mathrm{~km}$ along the line of sight between the ground receiver and the satellite (altitude $=20,200 \mathrm{~km}$ ). Each GPS receiver receives signals from four GPS satellites, 01, 22, 25, and 29, during the plotted interval.

NNE to SSW. The western edge of these images is covered by intense 630-nm airglow, which is probably due to the longitudinal gradient of electron density after the sunset. The sunset on the ground was at 1015 UT on August 4 and
6. The sky was clear only for the interval shown in Fig. 2. The 630-nm emission intensity at the zenith was $\sim 70 \mathrm{R}$ for 1330-1410 UT on August 4, 1999, and it decreased drastically from $\sim 250 \mathrm{R}(1133 \mathrm{UT})$ to $\sim 80 \mathrm{R}(1255-1403 \mathrm{UT})$ 

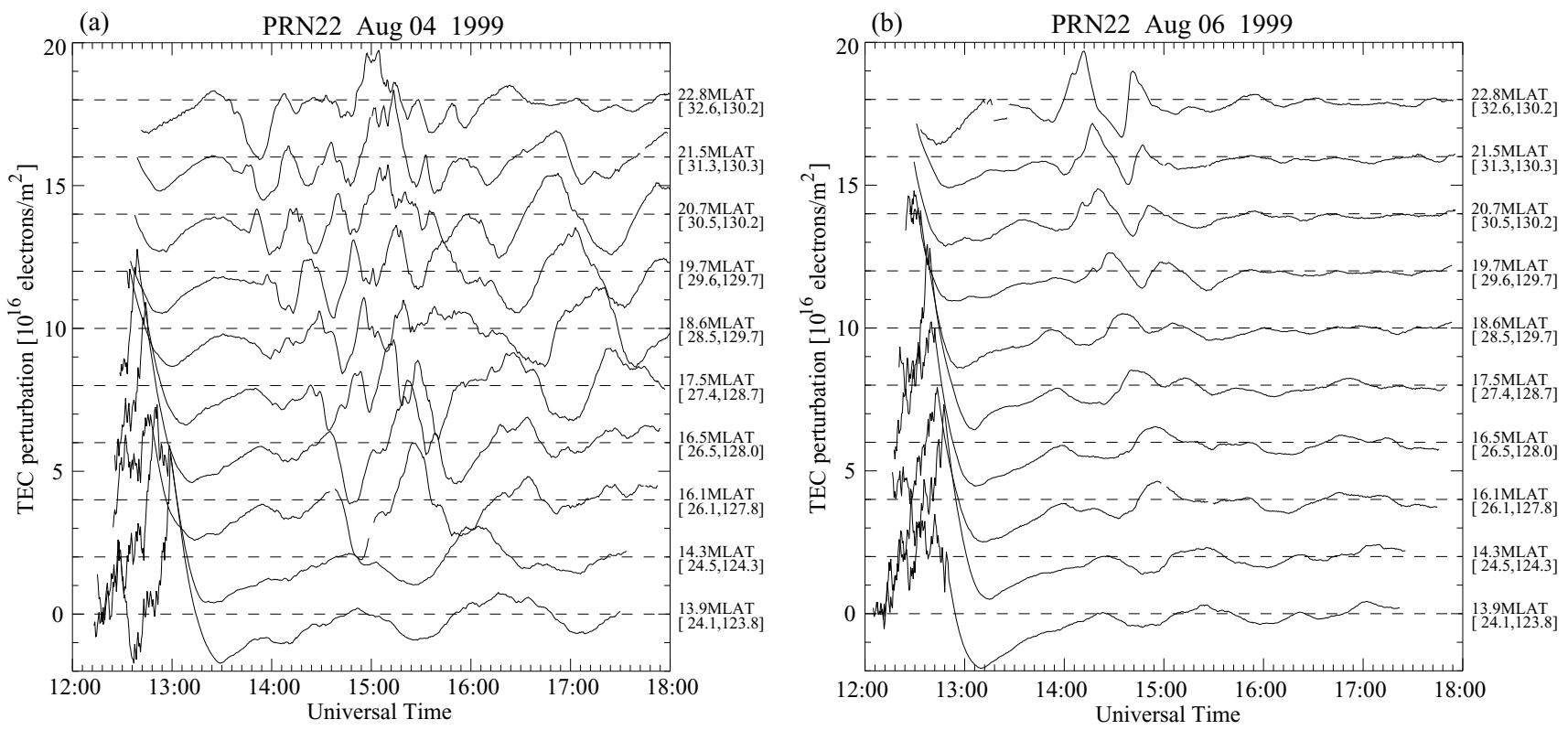

Fig. 4. TEC variations at $1200-1800$ UT (2030-0230 LT), obtained at several GPS receivers in Japan at geographic latitudes of $32.6^{\circ} \mathrm{N}-24.1^{\circ} \mathrm{N}$ $\left(22.8^{\circ}-13.9^{\circ}\right.$ MLAT) on (a) August 4, and (b) August 6, 1999. The TEC values are shown as differences from the running average over 2 hours. The GPS signal from satellite No. 22 was used to calculate the TEC values. Magnetic latitudes and geographic latitudes and longitudes of the GPS receivers are shown to the right of these panels.

on August 6, 1999.

A clear airglow depletion band, which is directed NWSE, was observed in the northeastern part of the images on August 4, 1999 (Fig. 2(a)). The band moved southwestward continuously with a velocity of $\sim 80 \mathrm{~m} / \mathrm{s}$. Note that other band structures were not seen in the south of this depletion band.

An intense airglow band was observed in southern Okinawa on August 6, 1999 (Fig. 2(b)). This east-west band slowly moved southward with a velocity of $\sim 40 \mathrm{~m} / \mathrm{s}$ with weakening the emission intensity. This band enhancement seems to correspond to the equatorial anomaly of the ionosphere. The slow equatorward motion of the band probably indicates that the anomaly was decreasing and going back to the south. It is noteworthy that no band structure was observed in northern Okinawa on this day.

\subsection{Measurements of TEC}

During the campaign, TEC was continuously measured by using more than 1000 GPS receivers over Japan by the Geographical Survey Institute, Japan. Figures 3(a) and 3(b) show maps of the TEC variations with a time interval of 15 minutes on August 4 and 6, 1999, respectively. The TEC values are shown as differences from the running average over 2 hours. This method is the same as that used by Saito et al. (1998). The TEC variations obtained are mapped on the geographical coordinates at an altitude of 250 $\mathrm{km}$ along the line of sight between the ground receiver and the GPS satellites (altitude $=20,200 \mathrm{~km}$ ). Note that some receivers are in the southern islands of Japan, including Okinawa $\left(26^{\circ} \mathrm{N}, 128^{\circ} \mathrm{E}\right)$.

On both August 4 and 6, medium-scale TIDs were observed in the mainland of Japan at geographic latitudes above $30^{\circ} \mathrm{N}$. The wave fronts are from northwest to southeast, and they travel southwestward with a typical velocity of $\sim 100 \mathrm{~m} / \mathrm{s}$. The wavelengths of these TIDs are $\sim 100-300$ $\mathrm{km}$. These features are similar to those reported in the literature (e.g., Mendillo et al., 1997; Taylor et al., 1998; Saito et al., 1998; Garcia et al., 2000; Saito et al., 2001; Kubota et al., 2000; Ogawa et al., 2002).

On August 4, 1999, the TID structures propagate to lower latitudes, below $30^{\circ}$. For example, a TEC enhancement at $\left(32^{\circ} \mathrm{N}, 130^{\circ} \mathrm{E}\right)$ at $1315 \mathrm{UT}$ propagates southwestward and reaches $\left(28^{\circ} \mathrm{N}, 128^{\circ} \mathrm{E}\right)$ at $1400 \mathrm{UT}$. This region is just north $(\sim 100 \mathrm{~km})$ of Okinawa and is well within the FOV of the all-sky imager. This enhanced TEC region probably corresponds to the weak enhancement of airglow just south of the depletion band in Fig. 2(a), and the airglow depletion band corresponds to the TEC depletion just north $\left(30^{\circ}-\right.$ $33^{\circ} \mathrm{N}, 128^{\circ} \mathrm{E}$ at $\left.1400 \mathrm{UT}\right)$ of this enhanced TEC. Ogawa et al. (2002) and Saito et al. (2001) have shown clear positive correspondence between the TEC and airglow enhancements in the medium-scale TIDs using data from the FRONT campaign. On August 6, 1999, the southward propagation of TID structures is not clear at latitudes below $30^{\circ} \mathrm{N}$.

To see the southward propagation of TIDs more clearly, we plotted the TEC variations detected by several GPS receivers as time series in Figs. 4(a) and (b). The magnetic latitudes of the receivers selected in these plots are from $13.9^{\circ}$ MLAT to $22.8^{\circ}$ MLAT, as shown at the right of each panel. The large TEC observed before 1300 UT below $18^{\circ}$ MLAT probably corresponds to the daytime high ionospheric electron density, though the time was just after the sunset.

The TIDs were detected as the wave-like structures with a period of 20-40 min at 1330-1800 UT on August 4, and 1400-1500 UT on August 6. Southward phase progression can be identified for several wave packets on both days. The TIDs propagate southward down to $16^{\circ}$ MLAT after 1330 

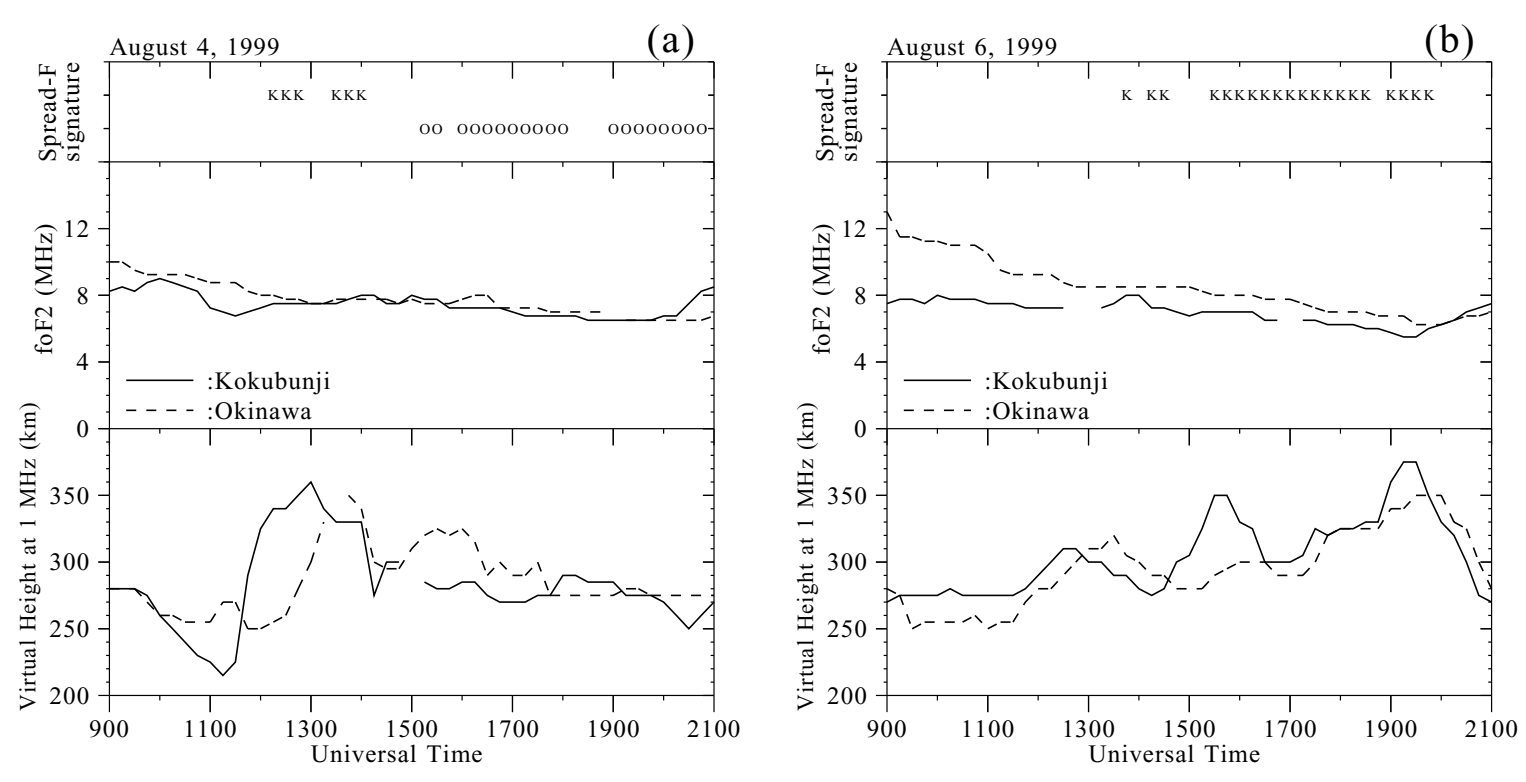

Fig. 5. Variations in the $F$-region virtual height at $1 \mathrm{MHz}, f_{o} F 2$ values, and spread-F signatures obtained at Kokubunji and Okinawa during the FRONT-2 campaign of (a) August 4, and (b) August 6, 1999. The locations of the stations are shown in Fig. 1.

UT on August 4, 1999, with a clear phase shift at around $18^{\circ}$ MLAT. On August 6 after 1400 UT, the wave packet seems to propagate down to $16^{\circ}$ MLAT, but the TID activity is much weaker than that of August 4. It is noteworthy that for both days, the TID activity decreases with decreasing magnetic latitudes from $23^{\circ}$ MLAT to $14^{\circ}$ MLAT.

\subsection{Ionosonde measurements}

To check the background condition of the ionosphere, we investigated ionosonde data obtained at Kokubunji and Okinawa during the campaign period. Figure 5 shows the virtual height at $1 \mathrm{MHz}, f o F 2$, and occurrence of spread-F observed at these stations at 0900-2100 UT on August 4 and 6, 1999. The foF 2 value decreases in these plots from 0900 UT (1730 $\mathrm{LT})$ to $2100 \mathrm{UT}(0530 \mathrm{LT})$, indicating nighttime recombination of ionospheric electrons. A sudden increase in $F$ layer height was observed at both Kokubunji and Okinawa at 1100-1300 UT on August 4. The airglow observation in Fig. 2(a) started at 1330 UT just after the $F$ layer rose. The difference in $f_{o} F 2$ between Okinawa and Kokubunji was higher on August 6 than on August 4. Spread-F signature was not observed at Okinawa on August 6.

\section{Summary and Discussion}

The FRONT-2 campaign was carried out to detect the equatorward edge of the southwestward-moving TIDs. During the campaign period of August 4-15, 1999, two nights were available for airglow measurements at Okinawa $\left(17.0^{\circ}\right.$ MLAT). The airglow and TEC features observed on these days are summarized as follows.

1. August 4, 1999: Medium-scale TIDs were observed as band structures in the TEC map in the mainland of Japan (wave front: NW-SE, propagation: southwestward). They came down to the north of the Okinawa latitude and were observed as a depletion band of the 630-nm airglow only in the northern sky of Okinawa at 2200-2230 LT.

2. August 6, 1999: Medium-scale TIDs were weakly ob- served as band structures in the TEC map in the mainland of Japan. The TID structures were not observed in the airglow images at Okinawa at 2000-2230 LT. A 630-nm airglow enhancement was observed in southern Okinawa.

3. For both days, the TID activity seen in the TEC data is weaken as the magnetic latitude decreases from $23^{\circ}$ MLAT to $14^{\circ}$ MLAT.

The medium-scale TIDs observed by the GPS-TEC network above the mainland of Japan $\left(\sim 21^{\circ}-36^{\circ}\right.$ MLAT $)$ were quite similar to those reported by Mendillo et al. (1997) and Garcia et al. (2000) based on the 630-nm airglow observations at Arecibo (28.8 $8^{\circ}$ MLAT). The TID activities apparently weaken as the latitude decreases from $23^{\circ}$ MLAT to $14^{\circ}$ MLAT (Fig. 4). Although the mechanism by which the medium-scale TIDs are generated has not been identified yet, these results suggest that there is some possible limit of the TID propagation at $\sim 18^{\circ}$ MLAT.

The airglow enhancement in southern Okinawa is likely due to the enhancement of the equatorial anomaly of ionospheric electron density. It may be related to the relatively high $K p$ value (4-) on this day. The eastward electric field associated with magnetic activities can cause uplifting of the equatorial ionosphere, which results in expansion of the equatorial anomaly to higher latitudes (e.g., Hanumath Sastri et al., 1992; Sobral et al., 1997; Shiokawa et al., 2000b). However, magnetic field data on this day indicates only a small substorm occurred at 1030-1200 UT. It is not clear why the anomaly came up to the Okinawa latitudes on August 6.

When the anomaly comes near the Okinawa latitudes, the electron density becomes high. The high electron (and ion) density may prevent gravity wave propagation through the ion drag, because the time constant of ion drag $t_{i}$ is given by $t_{i}=N /\left(N_{e} v\right)$, where $N, N_{e}$, and $v$ are the neutral and ion densities, and the collision frequency of an ion with neutral particles, respectively. Assuming $N=1.0 \times 10^{15} \mathrm{~m}^{-3}$, 
$N_{e}=1.0 \times 10^{12} \mathrm{~m}^{-3}$, and $v=0.5 \mathrm{~s}^{-1}$ (altitude $\sim 300$ $\mathrm{km})$, the value of $t_{i}$ becomes $2000 \mathrm{~s}$ (33 min), which is comparable to the observed wave period. Though the value of $N_{e}=1.0 \times 10^{12} \mathrm{~m}^{-3}$ is rather high for the nighttime ionospheric electron density, the anomaly possibly enhances the electron density in southern Okinawa. Actually the observed foF2 at Okinawa at $1400 \mathrm{UT}$ was $8.5 \mathrm{MHz}$, as shown in Fig. 5(b). This value corresponds to the peak electron density of $0.9 \times 10^{12} \mathrm{~m}^{-3}\left(=1.24 \times 10^{10}[f(\mathrm{MHz})]^{2}\right)$. Thus, when the anomaly comes up to Okinawa, the gravity waves, which can be a possible cause of TIDs, are easily declined through the ion drag.

The other possible cause of weakened TIDs at the Okinawa latitudes is the difference in atmospheric gravity wave activity. Because the mainland of Japan is covered by high mountains and the latitudes around Okinawa are mostly ocean, gravity wave generation may be more active above the mainland of Japan, affecting the generation of TIDs in the upper atmosphere.

It has been suggested that the medium-scale TIDs are generated by the interaction of gravity waves with the ionized atmosphere as the Perkins instability (Perkins, 1973). Thus, the latitudinal difference in magnetic dip angle may also affect the generation of TIDs. The growth rate of the instability is proportional to $\sin ^{2}(I)$, where $I$ is the magnetic dip angle. For dip angles of $49^{\circ}$ (mainland of Japan) and $38^{\circ}$ (Okinawa), the values of $\sin ^{2}(I)$ becomes 0.57 and 0.38 . This may affect the difference of the TID generation at different latitudes. As discussed by Garcia et al. (2000), however, the growth rate of the linear Perkins instability is usually not enough to generate the observed TID structures. Further observations of the latitudinal difference in TID characteristics will be important to investigating the generation and propagation of medium-scale TIDs.

Acknowledgments. We are grateful to Mr. M. Miyasato (Director) and the staff of the Kitaguni Elementary and Junior High School, Hedo, Okinawa, and the staff of the Okinawa Radio Observatory of the Communications Research Laboratory, for their kind support on our airglow observation during the campaign. Y. Katoh, T. Katoh, and M. Satoh of the Solar-Terrestrial Environment Laboratory, Nagoya University, are gratefully acknowledged for their kind support in the development of the all-sky imager system and the insulated boxes to keep it outside. This work was supported by Grant-in-Aid of the Ministry of Education, Culture, Sports, Science, and Technology of Japan (11440145).

\section{References}

Francis, S. H., A theory of medium-scale traveling ionospheric distur- bances, J. Geophys. Res., 79, 5245-5260, 1974.

Garcia, F. J., M. C. Kelley, J. J. Makela, and C.-S. Huang, Airglow observations of mesoscale low-velocity traveling ionospheric disturbances at midlatitudes, J. Geophys. Res., 105, 18,407-18,415, 2000.

Hamza, A. M., Perkins instability revisited, J. Geophys. Res., 104, 22,56722,575, 1999.

Hanumath Sastri, J., K. B. Ramesh, and D. Karunakaran, On the nature of substorm-related transient electric field disturbances in the equatorial ionosphere, Planet. Space Sci., 40, 95-103, 1992.

Hines, C. O., Internal atmospheric gravity waves at ionospheric heights, Can. J. Phys., 38, 1441-1481, 1964.

Hooke, W. H., Ionospheric irregularities produced by internal atmospheric gravity waves, J. Atmos. Terr. Phys., 30, 795-823, 1968.

Kubota, M., K. Shiokawa, M. K. Ejiri, Y. Otsuka, T. Ogawa, T. Sakanoi, H. Fukunishi, M. Yamamoto, S. Fukao, and A. Saito, Traveling ionospheric disturbances observed in the OI 630-nm nightglow images over Japan by using a multi-point imager network during the FRONT campaign, Geophys. Res. Lett., 24, 4037-4040, 2000.

Mendillo, M., J. Baumgardner, D. Nottingham, J. Aarons, B. Reinisch, J. Scali, and M. Kelley, Investigations of thermospheric-ionospheric dynamics with 6300-A images from the Arecibo Observatory, J. Geophys. Res., 102, 7331-7343, 1997.

Ogawa, T., N. Balan, Y. Otsuka, K. Shiokawa, C. Ihara, T. Shimomai, and A. Saito, Observations and modeling of $630 \mathrm{~nm}$ airglow and total electron content associated with traveling ionospheric disturbances over Shigaraki, Japan, Earth Planets Space, 54, this issue, 45-56, 2002.

Perkins, F., Spread F and ionospheric currents, J. Geophys. Res., 78, 218226, 1973.

Saito, A., S. Fukao, and S. Miyazaki, High resolution mapping of TEC perturbations with the GSI GPS network over Japan, Geophys. Res. Lett., 25, 3079-3082, 1998.

Saito, A., M. Nishimura, M. Yamamoto, M. Kubota, K. Shiokawa, Y. Otsuka, T. Tsugawa, S. Fukao, T. Ogawa, M. Ishii, T. Sakanoi, and S. Miyazaki, Traveling ionospheric disturbances detected in the FRONT campaign, Geophys. Res. Lett., 28, 689-692, 2001.

Shiokawa, K., Y. Katoh, M. Satoh, M. K. Ejiri, T. Ogawa, T. Nakamura, T. Tsuda, and R. H. Wiens, Development of optical mesosphere thermosphere imagers (OMTI), Earth Planets Space, 51, 887-896, 1999.

Shiokawa, K., Y. Katoh, M. Satoh, M. K. Ejiri, and T. Ogawa, Integratingsphere calibration of all-sky cameras for nightglow measurements, $A d v$. Space Sci., 26, 1025-1028, 2000a.

Shiokawa, K., Y. Otsuka, T. Ogawa, K. Igarashi, S. Miyazaki, F. J. Rich, A. Saito, and K. Yumoto, Comprehensive imaging observations of midlatitude ionospheric disturbances during storm time substorms, J. Geophys. Res., 105, 27,067-27,080, 2000b.

Sobral, J. H. A., M. A. Abdu, W. D. González, B. T. Tsurutani, I. S. Batista, and A. L. Clua de González, Effects of intense storms and substorms on the equatorial ionosphere/thermosphere system in the American sector from ground-based and satellite data, J. Geophys. Res., 102, 14,30514,313, 1997.

Taylor, M. J., J.-M. Jahn, S. Fukao, and A. Saito, Possible evidence of gravity wave coupling into the mid-latitude $\mathrm{F}$ region ionosphere during the SEEK campaign, Geophys. Res. Lett., 25, 1801-1804, 1998.

K. Shiokawa (e-mail: shiokawa@stelab.nagoya-u.ac.jp), Y. Otsuka, M. K. Ejiri, Y. Sahai, T. Kadota, C. Ihara, T. Ogawa, K. Igarashi, S. Miyazaki, and A. Saito 